\title{
SEQUENTIAL THORACIC METASTASECTOMY PROLONGS SURVIVAL BY RE-ESTABLISHING LOCAL CONTROL WITHIN THE CHEST
}

Michael T. Jaklitsch, MDa

Carlos M. Mery, MD

Jeanne M. Lukanich, MD

William G. Richards, $\mathrm{PhD}^{\mathrm{a}}$

Raphael Bueno, MD

Scott J. Swanson, MD

Steven J. Mentzer, MD

Ben D. Davis, MD

Elizabeth N. Allred ${ }^{\mathrm{b}}$

David J. Sugarbaker, MD
Objective: The value of sequential thoracic metastasectomies is unknown. We evaluate repeat metastasectomy for limited recurrences within the thorax.

Methods: From July 1988 to September 1998, 54 patients underwent 2 to 6 separate sequential procedures to excise metastases after recurrence isolated to the thorax. Kaplan-Meier survival and Cox modeling determined prognostic variables.

Results: Thirty-three men and 21 women, 22 to 76 years underwent $2(100 \%$, $\mathrm{n}=54), 3(50 \%), 4(22 \%)$, or 5 to $6(11 \%)$ metastasectomies. Fifty-four percent of patients had carcinoma, 35\% sarcoma, 9\% germ cell, and $2 \%$ melanoma. There were no operative deaths; all late deaths occurred from cancer. Median follow-up was 48 months. Cumulative 5-year survival from the second procedure was $57 \%$. After the second, third, fourth, and fifth procedures, respectively, permanent control was achieved in 15 (27\%) of 54 patients, $5(19 \%)$ of $27,1(8 \%)$ of 12 , and 0 of 7 . Recurrence amenable to additional surgery occurred in $27(50 \%)$ of 54, 12 (44\%) of 27, $6(50 \%)$ of 12 , and $1(17 \%)$ of 6 . Mean hazard for the development of unresectable recurrence increased from 0.21 after the second procedure to 0.91 after the fifth procedure. The 5-year survival for the 27 patients undergoing only 2 metastasectomies was $60 \%$ (median not yet reached), $33 \%$ for the 15 patients undergoing only 3 metastasectomies (median 34.7 months), and $38 \%$ for the 12 patients undergoing 4 or more (median 45.6 months). From the time a recurrence was declared unresectable, patients had a $19 \% 2$-year survival (median 8 months).

Conclusions: Multiple attempts to re-establish intrathoracic control of metastatic disease is justified in carefully selected patients, but the magnitude of benefit decays with each subsequent attempt. (J Thorac Cardiovasc Surg 2001;121:657-67)
Pusing ulmonary metastasectomy, or the surgical removal of metastases to the lung, is now accepted as an effective means of establishing local control in properly selected cases. Indications for the procedure include

From the Division of Thoracic Surgery, ${ }^{\mathrm{a}}$ Brigham and Women's Hospital, Dana-Farber Cancer Center, Harvard Medical School, and the Department of Neurology, ${ }^{\mathrm{b}}$ Children's Hospital, Harvard School of Public Health, Boston, Mass.

Read at the Twenty-fifth Annual Meeting of The Western Thoracic Surgical Association, Olympic Valley (Lake Tahoe), Calif, June 23-26, 1999.

Copyright () 2001 by The American Association for Thoracic Surgery

$0022-5223 / 2001 \$ 35.00+0 \quad \mathbf{1 2 / 6 / 1 1 2 8 2 2}$

doi: $10.1067 / \mathrm{mtc} .2001 .112822$ control of the primary site, no other distant metastatic disease, a technically feasible operation, and adequate cardiopulmonary reserve of the patient. ${ }^{1-3}$

Although no randomized trial has established a survival advantage of pulmonary metastasectomy, ${ }^{4}$ the overall 30\% 5-year survival is better than that of nonsurgical therapies. ${ }^{1-3}$ However, some authors argue that the careful selection of these patients explains the longterm survival and the value of pulmonary metastasectomy is unproven..$^{5-7}$

The potential role for repeat metastasectomy has not been as critically evaluated. ${ }^{2,3,8-10}$ In the report from the International Registry of Lung Metastases, 53\% of the 5206 cases had a recurrence after an initial pulmonary metastasectomy. ${ }^{11}$ In those 1042 patients able to under- 
go a second pulmonary metastasectomy, 5-year survival was $44 \%$ and 10-year survival was $29 \%$. Further analysis of this subgroup is not available.

We sought to address the potential role for repeated application of surgical resection of thoracic metastases for those unusual patients in whom the recurrence is restricted to the chest. Specifically, is there a limit to the number of attempts of thoracic metastasectomy conferring benefit on the patient? What factors establish that limit? What advice can be given to a patient facing a third or fourth attempt at thoracic metastasectomy in regard to risk and expected benefit?

Since 1988, the Division of Thoracic Surgery at Brigham and Women's Hospital has offered surgical resection of pulmonary metastases for those highly selected patients who continue to meet the basic criteria, regardless of the number of previous attempts. Repeat metastasectomy was offered to those patients with adequate cardiopulmonary reserve who had no cancer in any other sites and in whom an operation was technically feasible. Analysis of this cohort is used to address the aforementioned questions.

\section{Patients and methods}

Standardized data collection forms are prospectively completed for all patients evaluated by the Division of Thoracic Surgery at the Brigham and Women's Hospital at preoperative, operative, and postoperative interaction points. A fulltime thoracic surgical nurse/data manager compiles them into a computerized database. Standard preoperative data include spirometry, exercise oximetry, smoking history, and Karnofsky performance scale. Operative data include type of operation, surgical incisions, number and location of specimens, and intraoperative complications. Postoperative data include length of hospital stay and postoperative morbidity.

To be included in this series, the patient had to have had a repeat (second to sixth) metastasectomy within the past 10 years, independent of the time of the previous metastasectomy and primary resection attempts. Patients who had 1 or more metastasectomies and/or primary resection at other institutions more than 10 years ago and who then had a repeat effort to remove all intrathoracic metastases at our institution were included. No patients were excluded who had a repeat metastasectomy attempt within the past 10 years.

Indications for attempted repeat metastasectomy included previous resection and ongoing control of the primary site, absence of extrathoracic metastases at the time of considering repeat thoracic surgery, and adequate cardiopulmonary reserve as estimated by a predicted postoperative forced expiratory volume in 1 second of greater than $800 \mathrm{~mL}$ and a myocardial ejection fraction of greater than $30 \%$.

Resections of pulmonary metastases by video-assisted thoracic surgery (VATS) techniques have been performed by our group when considered technically feasible by the operating surgeon. As our experience with the technique has grown, we have no longer considered a repeat thoracic surgical procedure on a previously treated hemithorax as a contraindication. However, adhesions might preclude a VATS approach and lead to a conversion to open thoracotomy. When considering a VATS approach to metastasectomy, we obtain a high-resolution spiral computed tomographic (CT) scan to detect additional occult metastases. If all the nodules targeted for metastasectomy are physically located where they can be removed with a VATS technique, we generally choose to start exploration with a thoracoscope. If the nodule is found to be unresectable with a safe margin by a VATS technique, we convert to a thoracotomy. We did not prospectively collect data on the VATS conversion rate.

Staged thoracotomies up to 8 weeks apart for bilateral disease were considered a single attempt to locally control disease within the lungs (ie, a single repeat metastasectomy). A repeat attempt was defined as an additional operation to remove all recurrent cancer after a disease-free interval following the last surgical procedure. This did not include any operations that excised only benign nodules. Patients were considered to have unresectable disease if there was evidence of untreatable extrathoracic metastases when an additional thoracic operation was being considered, inadequate cardiopulmonary reserve, or technical inability to remove the recurrent disease. An incomplete resection was defined as an operation that did not remove all known tumor or one that left positive microscopic margins.

Operative data included the type of incision used, number of metastases removed, size of the largest metastases removed, magnitude of pulmonary resection (wedge versus lobe), location of metastases, and reasons for inability to remove all tumor. Operative mortality included all in-hospital deaths and deaths after discharge if they occurred within the first 30 days postoperatively.

The surgical nurse/data manager collected postoperative morbidity data on a daily basis. Over 100 precoded, adverse events were tracked by reviewing the inpatient record and interviewing resident, nursing, and attending staff. Complications were recorded in a prospective fashion and reviewed at a weekly quality assurance meeting of the division. Postoperative length of stay, final pathology results, and mortality were also recorded.

Follow-up was performed at postoperative clinic visits 1 week and 6 weeks after surgery. A repeat chest CT scan was generally performed 4 months after surgery and repeated every 4 months for the first 2 years, followed by every 6 months for the next 5 years. Radiographic evidence of recurrent disease was frequently re-evaluated with a repeat spiral chest CT scan in 6 to 12 weeks to judge the rate of progression and to look for additional signs of recurrence before additional surgery. Ninety-eight percent of survival data were actualized to October 1998.

Descriptive statistics included median, mean, and standard deviation. Univariate analysis of demographic variables between groups was performed with the Fisher exact test. There was a single case of repeat metastasectomy for melanoma. This case was not added to any of the other 3 histologic groups for univariate or multivariate analysis. 
Multivariate analysis of patient variables associated with survival was performed by Cox proportional hazards model. Survival curves were generated by Kaplan-Meier estimates.

To analyze the influence of antecedent history on the outcome of each repeated attempt at metastasectomy, we identified 4 separate "states" in which each patient could potentially dwell after a repeat metastasectomy: (1) no clinical evidence of recurrent disease, (2) recurrent disease amenable to repeat surgical metastasectomy, (3) recurrent disease not amenable to additional surgery or unresectable, and (4) death. We further identified the first 2 states (no clinical evidence of disease and recurrence amenable to additional surgery) as desirable states and the latter 2 (unresectable and death) as undesirable states. By this definition, all patients could potentially pass from a desirable state to an undesirable state, but no patient could pass back to a desirable state once they had reached unresectability or death. The denominator of each strata (metastasectomy attempt) is the number of patients in whom treatment failed at the previous stratum.

This stratification allowed us to perform sequential multivariate Cox proportional hazards models to examine the risk of passing to an undesirable state as a function of the number of previous metastasectomy attempts. This allows the time spent within each stratum to be a continuous variable and the risk of progressing to an undesirable state estimated for those at risk in the time interval. Average baseline hazards were calculated for each stratum based on the model. The survival curves for those remaining amenable to additional surgery were compared with those progressing to 1 of the 2 undesirable states with the log-rank test adjusted for the metastasectomy strata. ${ }^{12}$ The appendix contains a description of the models and variables used for the data analysis.

\section{Results}

Fifty-four patients had a repeat thoracic metastasectomy with curative intent between July 1988 and September 1998. The number of patients represents $14.6 \%$ of the 369 patients who underwent any type of thoracic metastasectomy during the same time period. Thirty-three of the patients undergoing repeat metastasectomy were male $(61 \%)$ and 21 were female. The median age was 54 years, with a range from 22 to 76 years.

All 54 patients had at least 2 thoracic metastasectomies in fulfillment of the entry criteria for this analysis. Twenty-seven $(50 \%)$ of the 54 patients had at least 3 thoracic metastasectomies, $12(22 \%)$ had at least 4 thoracic metastasectomies, $6(11 \%)$ had at least 5, and 1 patient had 6 thoracic metastasectomies. For statistical analysis, the 6 patients with 5 or more metastasectomies were combined into a single group.

As summarized in Table I, the metastatic cell type of 54 surgical specimens obtained at the first and second thoracic metastasectomies included 29 (54\%) carcino- mas, $19(35 \%)$ sarcomas, $5(9 \%)$ germ cell tumors, and $1(2 \%)$ melanoma. The 1 patient who underwent a sixth thoracic metastasectomy had a germ cell tumor. There was a significant statistical trend toward more sarcomas and germ cell tumors among patients undergoing more than 3 thoracic metastasectomies $(P=.0004)$.

A total of $117(76 \%)$ of the 154 operations performed on this cohort involved only pulmonary parenchymal resections, whereas $37(24 \%)$ operations involved extended thoracic metastasectomies including resections of diaphragm, pericardium, mediastinal nodes, and chest wall. Fourteen operations (9\%) combined pulmonary parenchymal and extended thoracic resections. Twenty-three $(15 \%)$ operations were extended thoracic resections of mediastinal and/or chest wall metastases without a pulmonary resection.

Surgical incisions included 1 clamshell incision, 5 staged bilateral thoracotomies, 17 median sternotomies, 94 unilateral thoracotomies, 7 thoracotomies with en bloc chest wall resection, 4 bilateral VATS procedures, and 26 unilateral VATS procedures. The largest resection performed was a pneumonectomy, which occurred $5 \%$ of the time. Lobectomy was performed $10 \%$ of the time, segmentectomy $4 \%$, wedge resection $62 \%$, chest wall resection $12 \%$, and a mediastinal resection $7 \%$.

Table I summarizes the important demographic variables of the 54 patients grouped by the maximum number of metastasectomies. The increase in the number of men and the decrease in age at both the primary procedure and current procedure of the group undergoing 5 or 6 metastasectomies reflects the dominance of germ cell histologic type (77\% within this group). Furthermore, the increase in the size of the largest metastasis resected likely reflects a willingness to resect larger recurrences in younger patients. There was no difference in the use of adjuvant therapy for the primary tumor as a function of increasing attempts to perform thoracic metastasectomies, but there appeared to be a trend toward increasing use of adjuvant therapy with increasing attempts at surgery. This trend did not reach statistical significance. There was likewise no difference in the mean disease-free interval from the last procedure, the median number of metastases removed, the size of the largest metastasis removed, nor the use of wedge resection alone.

A VATS technique was used in 11 of the primary metastasectomies, with an average of 1.6 nodules (range 1-4 nodules) being removed. When these 11 patients had a repeat metastasectomy, 4 had a repeat ipsilateral VATS resection, 3 had an ipsilateral thoracotomy, 3 had a contralateral thoracotomy, and 1 had a contralateral VATS resection. 
Table I. Cross-sectional characteristics of metastasectomy strata

\begin{tabular}{|c|c|c|c|c|}
\hline & \multicolumn{4}{|c|}{ Metastasectomy attempt } \\
\hline & $2(n=54)$ & $3(n=27)$ & $4(n=12)$ & $5(n=6)$ \\
\hline Male, n (\%) & $33(61)$ & $21(78)$ & $10(83)$ & $6(100)$ \\
\hline Age at this metastasectomy (median) & 52.9 & 51.5 & 44.5 & 33 \\
\hline Age at primary metastasectomy (median) & 47.7 & 44.8 & 34.6 & 27.8 \\
\hline \multicolumn{5}{|l|}{ Histologic type at this procedure, $\mathrm{n}(\%)$} \\
\hline Carcinoma & $29(54)$ & $9(33)$ & $2(17)$ & $1(17)$ \\
\hline Sarcoma & $19(35)$ & $14(52)$ & $6(50)$ & $1(17)$ \\
\hline Germ cell & $5(9)$ & $4(15)$ & $4(33)$ & $4(67)$ \\
\hline Melanoma & $1(2)$ & 0 & 0 & 0 \\
\hline Chemotherapy for primary, $\mathrm{n}(\%)$ & $14(26)$ & $8(30)$ & $5(42)$ & $5(83)$ \\
\hline Radiation therapy for primary, $\mathrm{n}(\%)$ & $16(30)$ & $9(33)$ & $5(42)$ & $2(33)$ \\
\hline Disease-free interval from last metastasectomy (mean $\pm \mathrm{SD})$ & $21.3 \pm 18$ & $19.7 \pm 23$ & $28.3 \pm 72$ & $19.3 \pm 17$ \\
\hline No. metastases (median) & 1.0 & 2.0 & 2.0 & 2.0 \\
\hline Size of largest metastasis, $\mathrm{cm}$ (median) & 2.5 & 3.0 & 3.2 & 4.3 \\
\hline Wedge, $\mathrm{n}(\%)$ & $36(67)$ & $17(63)$ & $7(58)$ & $4(67)$ \\
\hline VATS, n $(\%)$ & $16(30)$ & $4(15)$ & $1(8)$ & 0 \\
\hline Chemotherapy this metastasectomy, $\mathrm{n}(\%)$ & $10(18)$ & $5(18)$ & $4(33)$ & $1(17)$ \\
\hline Radiation therapy this metastasectomy, $\mathrm{n}(\%)$ & $4(7)$ & $5(18)$ & $3(25)$ & $2(33)$ \\
\hline
\end{tabular}

Distribution of the cell type of the metastases removed within each group of metastasectomy attempt. There was a significant statistical trend toward more sarcomas and germ cell tumors among patients undergoing more than 3 thoracic metastasectomies $(P=.0004)$.

Table II. Hazard of progressing to an undesirable outcome as a function of metastasectomy attempt

\begin{tabular}{|c|c|c|c|c|c|}
\hline \multirow[b]{2}{*}{ Total procedures } & \multicolumn{2}{|c|}{ Desirable } & \multicolumn{2}{|c|}{ Undesirable } & \multirow[b]{2}{*}{$\begin{array}{c}\text { HR of progressing } \\
\text { to undesirable } \\
\text { Mean } \pm S D\end{array}$} \\
\hline & $\begin{array}{c}\text { No } \\
\text { recurrence } \\
n(\%)\end{array}$ & $\begin{array}{c}\text { Resectable } \\
\text { recurrence } \\
n(\%)\end{array}$ & $\begin{array}{c}\text { Unresectable } \\
\text { recurrence } \\
n(\%)\end{array}$ & $\begin{array}{l}\text { Death } \\
n(\%)\end{array}$ & \\
\hline Two & $15(27)$ & $27(50)$ & $3(6)$ & $9(17)$ & $0.21 \pm 0.16$ \\
\hline Three & $5(19)$ & $12(44)$ & $2(7)$ & $8(30)$ & $0.54 \pm 0.35$ \\
\hline Four & $1(8)$ & $6(50)$ & $2(17)$ & $3(25)$ & $0.86 \pm 0.19$ \\
\hline Five or more & 0 & $1(17)$ & $2(33)$ & $3(50)$ & $0.91 \pm 0.14$ \\
\hline
\end{tabular}

Each metastasectomy attempt had 1 of 4 possible outcomes: No recurrence of disease to date, a temporary period of disease control followed by intrathoracic recurrence amenable to additional surgery to re-establish control within the chest, a temporary period of disease control followed by an unresectable recurrence, or death. The former 2 states are considered "desirable" and the latter 2, "undesirable." The number of patients in the state of recurrence amenable to more surgery became the denominator of the next metastasectomy attempt. The hazard ratio $(H R)$ of progressing to an undesirable outcome as a function of metastasectomy attempt is summarized to the right of the table.

Fourteen patients had a VATS resection at the second metastasectomy, with an average of 1.5 nodules (range 1-6 nodules) being removed. Four patients had a VATS resection at the third metastasectomy, with an average of 1.5 nodules (range 1-2) being removed. One patient had a VATS resection at the fourth metastasectomy, with 2 nodules being removed. Of the 21 patients having a VATS resection at the second or greater metastasectomy, 7 (33\%) had had previous ipsilateral VATS resections, 5 (24\%) had had previous ipsilateral open resections, and $43 \%$ had had only contralateral procedures. The largest nodule removed at the time of VATS resection was an average of $1.3 \mathrm{~cm}$ (range $0.2-4.5 \mathrm{~cm}$ ).

Thirty-seven percent of these patients received no adjuvant therapy. Twenty percent received chemothera- py only, $13 \%$ received radiation only, and $30 \%$ received a combination of radiation and chemotherapy.

The median number of metastases excised at each operation was 1 for first metastasectomy (range 1-6), 1 for second (range 1-20), 2 for third (range 1-10), 2 for fourth (range 1-9), and 2 for 5 or more metastasectomies (range 1-8). No statistically significant difference existed among these groups.

The median size of the largest metastasis excised at each operation was $1.7 \mathrm{~cm}$ for first metastasectomy (range 0.2-15 cm), $2.4 \mathrm{~cm}$ for second (range 0.1-12 $\mathrm{cm}), 3.2 \mathrm{~cm}$ for third (range $0.2-12.5 \mathrm{~cm}$ ), $2.9 \mathrm{~cm}$ for fourth (range $1-18 \mathrm{~cm}$ ), and $4.5 \mathrm{~cm}$ for fifth or more metastasectomies (range 2-20 cm). Size of largest metastasis was not a predictor of long-term survival. 
Table III. Recurrence after each procedure

\begin{tabular}{lc}
\hline Previous metastasectomies & $H R(95 \% \mathrm{CI})$ \\
\hline Two & Reference group \\
Three & $3.2(1.2,8.6)$ \\
Four & $5.5(1.8,17.4)$ \\
Five or more & $12.9(4.3,39)$
\end{tabular}

Hazards of developing recurrence as a function of the number of previous metastasectomies. The hazard ratios $(H R)$ significantly increase after each additional procedure. $C I$, Confidence intervals.

A complete resection was obtained in 141 (91.6\%) of 154 total operations. Of the 13 patients undergoing incomplete resections, 7 had an additional operation from 2 to 39 months later. Five of these 7 subsequently had a complete resection by way of a larger operation. The remaining 2 patients had an additional incomplete resection 12 and 13 months later.

Sequential follow-up to October 1998 was achieved for $53(98 \%)$ of the 54 patients. Median follow-up time is 48 months, measuring from the day of the second metastasectomy. Cumulative 5-year survival is 57\% from the time of the second metastasectomy. There were no operative deaths. All late deaths occurred from cancer.

Table II summarizes the distribution of 4 possible outcome states as a function of metastasectomy attempts: no recurrence of disease to date, a temporary period of disease control followed by intrathoracic recurrence amenable to additional surgery to re-establish control within the chest, a temporary period of disease control followed by an unresectable recurrence, or death. The former 2 states are considered "desirable" and the latter 2, "undesirable." The number of patients in the state of recurrence amenable to more surgery becomes the denominator of the next metastasectomy attempt. The hazard ratio of progressing to an undesirable outcome as a function of metastasectomy attempt is summarized to the right of the table. The probability of progressing to unresectability or death after a repeat metastasectomy increased from .21 after the second attempt to .91 after the fifth attempt.

Of the 33 patients who have had an unresectable recurrence, 18 (55\%) lacked sufficient cardiopulmonary reserve to undergo additional surgery, 11 (33\%) had technically unresectable disease, and 4 (12\%) had recurrences outside the thorax.

The 5-year survival from second metastasectomy (model 1; see appendix) for those undergoing 2 procedures is 59\% (median greater than 60 months), 33\% for those undergoing 3 procedures (median 34.7 months), and $38 \%$ for those undergoing 4 or more procedures (median 45.6 months). All deaths are due to recurrent
Table IV. Death after each procedure

\begin{tabular}{lc}
\hline Previous metastasectomies & $H R(95 \% \mathrm{CI})$ \\
\hline Two & Reference group \\
Three & $3.0(1.2,7.8)$ \\
Four & $2.6(0.7,9.7)$ \\
Five or more & $7.1(2.1,24)$ \\
\hline
\end{tabular}

Hazards of death as a function of the number of previous metastasectomies. Survival remains stable until the fifth procedure, when hazard ratios $(H R)$ significantly increase. $\mathrm{CI}$, Confidence intervals.

Table V. Progression to an undesirable state after each procedure

\begin{tabular}{lc}
\hline Previous metastasectomies & $H R(95 \% \mathrm{CI})$ \\
\hline Two & Reference group \\
Three & $2.7(1.2,6.3)$ \\
Four & $3.4(1.2,9.9)$ \\
Five or more & $8.5(3.0,24)$ \\
\hline
\end{tabular}

Hazards of progression to an undesirable state (ie, unresectability or death) as a function of the number of previous metastasectomies. Rate of progression slightly increases after the third and fourth procedures and significantly increases after the fifth attempt. $H R$, Hazard ratios; $C I$, confidence intervals.

unresectable cancer. The slope of decay of each survival curve is similar, suggesting a similar biology of the unresectable tumor between groups. Yet the re-establishment of local control in the chest has extended survival for an additional 1 to 3 years for those patients fortunate enough to undergo a third or fourth metastasectomy.

When comparing those patients who progressed to an undesirable state with those who remained within a desirable state, the Cox proportional hazards model (model 2; see appendix) gives a hazard ratio of 3.8 (95\% confidence intervals: $1.1,12.8$ ) for the undesirable cohort, meaning those patients progressing to an unresectable recurrence are 3.8 times more likely to die.

The above analysis is unable to differentiate whether the increase in survival with additional procedures is due to repeat surgery or to the patients being obligated to survive longer to experience a third or fourth procedure.

The number of metastasectomy attempts does not correlate with disease-free survival (model 3; see appendix). Predictors of disease-free survival include metastatic involvement of only the lung parenchyma compared with extended resections involving the chest wall or mediastinum $(P=.003)$. Other predictor variables not associated with long-term survival are listed in the appendix.

To determine whether thoracic disease tended to recur more frequently and sooner after each procedure, we constructed a new model (model 4; see appendix). The hazard ratios for recurrence increase dramatically with each procedure, from 3.2 after 3 metastasectomies 


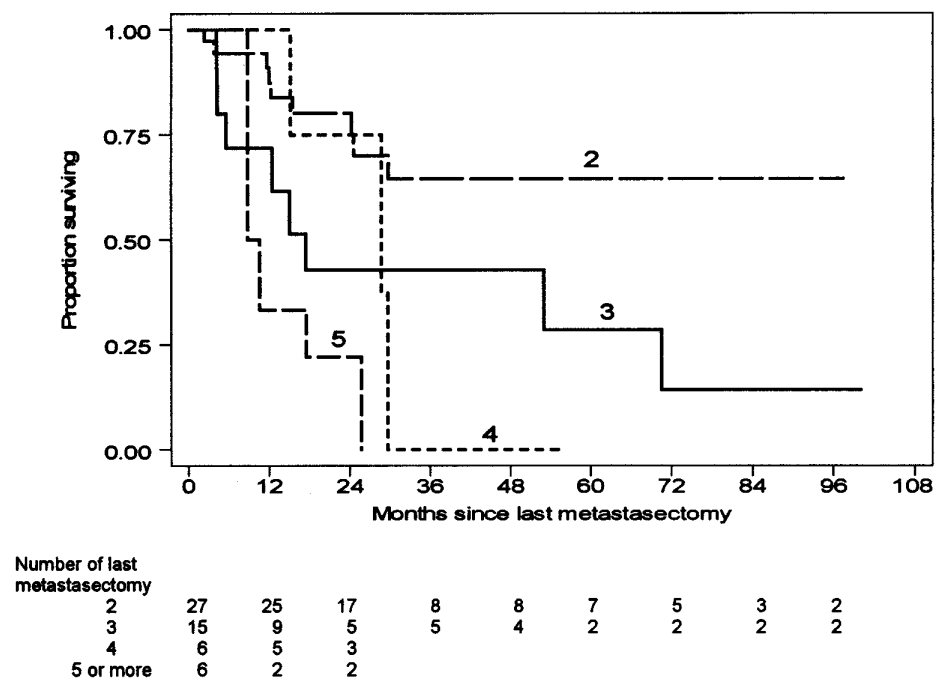

Fig 1. Kaplan-Meier survival curves from the time of the last metastasectomy for patients undergoing 2, 3, 4, or 5 or more procedures (model 5; see appendix). Patients undergoing several procedures are included in several curves. All deaths are due to cancer. The slopes of decay for the survival curves are fairly similar to each other until patients undergo their fifth procedure (see Table IV). The advantage of repeat metastasectomy in terms of survival can be thought of as the additional time that patients have had before undergoing the specific procedure that places them in each curve.

to 12.9 after 5 or more procedures (Table III). Although recurrence increases with each procedure, survival (model 5; see appendix) is fairly stable until the fifth or more procedure (Table IV, Fig 1).

Rate of progression to an undesirable state (ie, unresectable recurrence or death) (model 6; see appendix) slowly increases until the fifth or more procedure (Table V). Once patients were considered to have unresectable disease, survival was independent of the number of antecedent procedures (model 7; see appendix). The lower curve in Fig 2 shows the survival curve for these patients from the time of recognized unresectable recurrence. Median survival was only 8 months, with a 2 -year survival of $19 \%$. The upper curve in Fig 2 depicts the overall survival of the cohort, with a 5-year survival of $57 \%$. The advantage conferred by repeat metastasectomies lies somewhere between these 2 curves.

\section{Discussion}

The value of an aggressive surgical approach for patients with recurrence confined to the thorax despite previous metastasectomy was intuitive but not previously quantified. All patients within this study group met previously established criteria including control of all extrathoracic disease, adequate cardiopulmonary reserve, and a technically feasible operation to remove all recognized disease. The highly selected group pre- sented here enjoyed a cumulative 57\% 5-year survival through repeated operations to remove local recurrences confined to the chest. This benchmark compares favorably with the $30 \%$ to $48 \%$ cumulative 5-year survival for initial thoracic metastasectomy previously reported by numerous authors. ${ }^{1-3,8,13-15}$ Furthermore, there was a correlation between preserved intrathoracic local control and 5-year survival. Once local control had been lost, further survival fell dramatically to a median of 8 months and a 2-year survival of $19 \%$, regardless of the number of previous procedures.

The re-establishment of local control with multiple metastasectomy attempts contributed to extended survival. This confirms similar findings in large series. ${ }^{1,2}$ The median survival of 8 months for patients with unresectable recurrence correlates with previous observations that patients with incomplete resections or unresectable disease have median survivals of 6 to 12 months. ${ }^{2,4}$ The salvage rate of 5 of 13 incomplete resections with additional adjuvant therapy and surgery is encouraging.

Fourteen percent of our patients had extended intrathoracic resections, including mediastinal nodes, pericardium, diaphragm, and chest wall. Other authors have noted satisfactory results with similar extended resections. ${ }^{3,8,16}$ Although the need for an extended resection was a negative prognostic predictor of longterm clinical disease-free survival compared with lung 


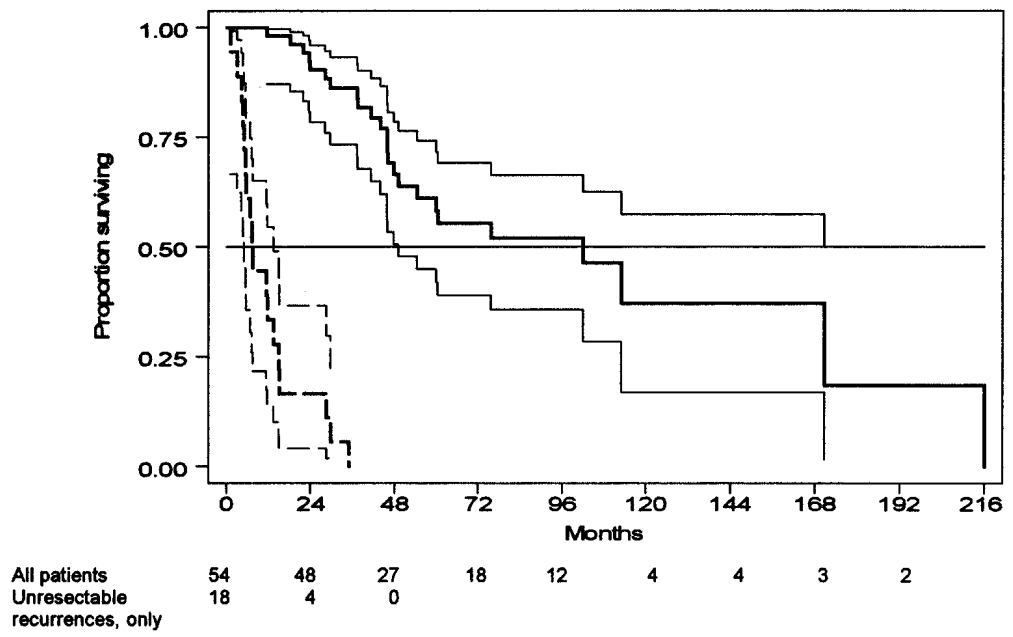

Fig 2. Kaplan-Meier survival curves providing limits for prolongation of survival with repeat metastasectomy. The lower curve depicts survival of patients once they have a recurrence not amenable to further surgery. Median survival is 8 months with a 2-year survival of $19 \%$ after the disease is declared unresectable. The upper curve shows the overall survival for the cohort from the time of the second procedure with a 5-year survival of 57\%. The advantage conferred by repeat metastasectomy, in terms of survival, lies somewhere between both curves.

resections alone, we do not believe it should be regarded as a contraindication to surgery since this subgroup enjoyed a 5-year survival of 30\% (median 47 months).

The percentage of patients undergoing repeat metastasectomy in this cohort corresponds well with the International Registry data of 5206 patients. In that report, $15 \%$ of patients underwent a second metastasectomy, $4 \%$ underwent a third, and $1 \%$ had 4 or more with a maximum of 7 metastasectomies. ${ }^{11}$ However, the survival of our selected group is better than that of the reported survival in Pastorino's report, ${ }^{11}$ which was only $36 \%$ at 5 years and $26 \%$ at 10 years with a median of 35 months. This difference may be due to the number of germ cell tumors within our group or to the improved predictive power of their larger, multi-institutional registry. Alternatively, this may reflect a benefit of improved survival and local control provided by repeated operations for thoracic recurrence.

A similar cohort to our study group was reported by Robert and associates. ${ }^{10}$ Of 276 patients undergoing metastasectomy, 63 had a second-stage metastasectomy, 12 a third-stage, and 2 patients a fourth-stage. Median follow-up of this cohort was 38 months, with a 2-year survival of $69 \%$ and a 5-year survival of $48 \%$. The association between preservation of intrathoracic local control and survival was not tested, but the survival curves for patients having first- and second-stage metastasectomy appear similar to each other. Our analysis combined with this previous report suggests that each successful metastasectomy re-establishes the probability for long-term survival by returning the patient to a clinical local control state.

The patients within this cohort were carefully selected. The $92 \%$ complete resection rate compares favorably with 2 previous reports of $85 \% 2,17$ and likely reflects the careful selection of patients with resectable disease for additional attempts at metastasectomy. Most authors agree that surgery is indicated if it is technically feasible to remove all tumor while preserving adequate cardiopulmonary function. ${ }^{12,18}$ We would extend the indications to include those rare patients who have a recurrence confined to the thorax, independent of the number of previous surgical excisions. On the other hand, the probability of benefiting from a repeated attempt at thoracic metastasectomy diminishes after each previous operation. Additional attempts should be carefully considered.

Chest recurrence increases after each additional metastasectomy. These results suggest that either the tumor biology is changing or the host-tumor interaction begins to favor the tumor. However, survival is relatively stable until the fifth procedure. This "stability" of survival curves irrespective of the increase in recurrence rates may be due to the re-establishment of local control in the chest.

Some authors remain skeptical about the value of thoracic metastasectomy. ${ }^{6,19}$ These authors point out that the larger series are heterogeneous populations with mixed histologic types and mixed doubling times. Several series 
have correlated the best long-term survival with extended disease-free interval ${ }^{12}$ or long tumor doubling times. They argue that patients with a long tumor doubling time, smaller tumor burden, and favorable histologic type will do well whether they have a metastasectomy or not. If this population makes up $30 \%$ to $40 \%$ of a given series, these selection factors may translate into a 30\% 5-year survival without any benefit from surgery. These criticisms have generally been put to rest by modern studies that have demonstrated a survival advantage of surgery compared with nonsurgical treatment. ${ }^{1,20}$

Our analysis argues against their point of view because the subgroup that eventually lost intrathoracic local control serves as its own control. Before the unresectable recurrence, these patients had a median survival of 48 months. Expected survival drops to 8 months after the recurrence that can no longer be removed. Furthermore, we believe the $57 \%$ cumulative 5-year survival of our entire study group (twice that of previous reports) is due to the prolongation of clinical disease-free status for as long as possible.

Other authors have failed to find a relationship between long-term survival and the number of metastases removed, disease-free interval, tumor doubling time, age, sex, location, diameter of metastases, previous chemotherapy, and extent of resection as long as a complete resection was achieved. . $^{2,5,7,8,15}$ The association of these variables with disease-free survival does not appear to be as strong as the association between survival and complete resection. ${ }^{7,18}$

One cannot hope to use a highly selected group to conclusively prove the value of repeat metastasectomy. However, this initial analysis is encouraging and seems to justify repeat metastasectomy for carefully selected patients. Part of this selection includes the repetition of imaging studies 6 weeks after documenting a recurrence. Patients with rapidly progressive disease or the development of numerous additional occult metastases would not have been offered repeat metastasectomy.

Further work is required to understand the value of repeat surgery for this biologically unique group of patients who continue to have recurrences but only within the chest. Data on similar subgroups of patients offered repeat metastasectomy should be sought from larger series of patients, including The International Registry of Lung Metastases. Furthermore, new treatment options are becoming available that might alter the need for additional surgery in the future. These include new adjuvant agents, including angiostatin agents that might alter the thoracic "soil" which facilitates the growth of these malignant seeds. Stereotactic radiation therapy for the chest is currently offered in a handful of centers and may become more universally applied. Finally, spiral CT scans and positron emission tomographic scanning may assist in more accurately judging the extent of small metastatic disease within the chest and facilitate surgical metastasectomy.

At present, we feel justified in offering an aggressive surgical approach to this minority of patients with recurrent disease confined only to the thorax. Few patients will meet these criteria. Once recurrent disease is recognized, a careful evaluation of heart and lung function should be undertaken. Extrathoracic disease should be sought. The imaging studies should be repeated in 6 weeks to exclude rapidly progressive disease and allow additional occult metastases to manifest themselves. However, if the patient has sufficient cardiopulmonary reserve and removal of all visualized disease remains technically feasible, repeat surgical metastasectomy should be offered.

Received for publication June 29, 1999; revisions requested Aug 23, 1999; final revisions received Oct 6, 2000; accepted for publication Nov 6, 2000.

Address for reprints: Michael T. Jaklitsch, MD, Division of Thoracic Surgery, Brigham and Women's Hospital, 75 Francis St, Boston, MA 02115.

\section{REFERENCES}

1. Marincola F, Mark J. Selection factors resulting in improved survival after surgical resection of tumors metastatic to the lungs. Arch Surg 1990;125:1387-93.

2. Girard P, Baldeyrou P, LeChevalier T, LeCesne A, Brigandi A, Grunenwald D. Surgery for pulmonary metastases. Who are the 10-year survivors? Cancer 1994;74:2791-7.

3. Martini N, McCormack P. Evolution of the surgical management of pulmonary metastases. Chest Surg Clin North Am 1998;8:13-27.

4. Pass H. Surgical management of pulmonary metastases. Curr Opin Oncol 1998;10:146-50.

5. Aberg T, Malmberg KA, Nilsson B, Nou E. The effect of metastasectomy: Fact or fiction? Ann Thorac Surg 1980;30:378-84.

6. Aberg T. Selection mechanisms as major determinants of survival after pulmonary metastasectomy. Ann Thorac Surg 1997;63:611-2.

7. Todd T. The surgical treatment of pulmonary metastases. Chest 1997;112:287S-90S.

8. McAfee MK, Allen MS, Trastek VF, Ilstrup DM, Deschamps C, Pairolero PC. Colorectal lung metastases: results of surgical excision. Ann Thorac Surg 1992;53:780-6.

9. Grunenwald D, Spaggiari L, Girard P, Baldeyrou P, Posea R, Lamer C, et al. Lung resection for recurrence after pneumonectomy for metastases. Bull Cancer 1997;84:277-81.

10. Robert JH, Ambrogi V, Mermillod B, Dahabreh D, Goldstraw P. Factors influencing long-term survival after lung metastasectomy. Ann Thorac Surg 1997;63:777-84.

11. Pastorino U, Buyse M, Friedel G, Ginsberg RJ, Girard P, Goldstraw P, et al. Long-term results of lung metastasectomy: 
prognostic analyses based on 5206 cases. J Thorac Cardiovasc Surg 1997;113:37-49.

12. Prentice RL, Williams BJ, Peterson AV. On the regression analysis of multivariate failure time data. Biometrika 1981;68:373-9.

13. van Geel AN, Pastorino U, Jauch KW, Judson IR, van Coevorden F, Buesa JM, et al. Surgical treatment of lung metastases: the European Organization for Research and Treatment of CancerSoft Tissue and Bone Sarcoma Group study of 255 patients. Cancer 1996;77:675-82.

14. Mountain C, McMurtrey M, Hermes K. Surgery for pulmonary metastases: a 20-year experience. Ann Thorac Surg 1984;38:323-30.

15. Patterson G, Todd TR, Ilves R, Pearson FG, Cooper JD. Surgical management of pulmonary metastases. Can J Surg 1982;25:102-5.
16. Putnam JB Jr, Suell DM, Natarajam G, Roth JA. Extended resection of pulmonary metastases: Is the risk justified? Ann Thorac Surg 1993;55:1440-6.

17. Roth JA, Pass PI, Wesley MN, White D, Putnam JB, Seipp C. Comparison of median sternotomy and thoracotomy for resection of pulmonary metastases in patients with adult soft-tissue sarcomas. Ann Thorac Surg 1986;42:134-8.

18. Putnam JB Jr, Roth JA. Prognostic indicators in patients with pulmonary metastases. Semin Surg Oncol 1990;6:291-6.

19. Casciato D, Nagurka C, Tabbarah H. Prolonged survival with unresected pulmonary metastases. Ann Thorac Surg 1983; 36:202-8.

20. Harpole DH Jr, Johnson CM, Wolfe WG, George SL, Siegler HF. Analysis of 945 cases of pulmonary metastatic melanoma. J Thorac Cardiovasc Surg 1992;103:743-50.

\section{Appendix: Cox proportional hazards models used for the analysis of sequential metastasectomies}

Model 1. Survival from second metastasectomy by number of procedures Time zero: Date of second metastasectomy

Outcome(s): Death

Censored event(s): End of follow-up

Strata: Number of metastasectomies performed in each patient

Model 2. Survival from second metastasectomy by attainment of undesirable state

Time zero: Date of second metastasectomy

Outcome(s): Death

Censored event(s): End of follow-up

Strata: Desirability state

Reference group: Patients that remained in desirable state

Model 3. Disease-free survival after each procedure

Time zero: Date of last metastasectomy

Outcome(s): Death, recurrence

Censored event(s): End of follow-up

Strata: Procedure (metastasectomy)

Reference group: Second metastasectomy

*Patients with several procedures are included in several strata

Model 4. Time to recurrence after each procedure

Time zero: Date of last metastasectomy

Outcome(s): Recurrence

Censored event(s): End of follow-up, death (without recurrence)

Strata: Procedure (metastasectomy)

Reference group: Second metastasectomy

*Patients with several procedures are included in several strata
Model 5. Survival after each procedure Time zero: Date of last metastasectomy Outcome(s): Death

Censored event(s): End of follow-up, going to next procedure Strata: Procedure (metastasectomy)

Reference group: Second metastasectomy

*Patients with several procedures are included in several strata

Model 6. Rate of transition to an undesirable state (unresectability or death)

Time zero: Date of last metastasectomy

Outcome(s): Unresectable metastasis, death

Censored event(s): End of follow-up, going to next procedure

Strata: Procedure (metastasectomy)

Reference group: Second metastasectomy

*Patients with several procedures are included in several strata

Model 7. Survival after unresectability

Time zero: Date of claimed unresectability

Outcome(s): Death

Censored event(s): End of follow-up

Strata: Number of metastasectomies performed in each patient

Reference group: Patients with 2 metastasectomies

*Only patients with unresectable disease $(\mathrm{n}=27)$ were included

Other variables included in the models and found not to be associated with survival or recurrence were sex, histology, disease-free intervals, history of chemotherapy or radiation therapy for the primary tumor, number and size of metastases resected at current metastasectomy, site of disease, wedge versus larger resections, completeness of resection, use of VATS incision, and history of chemotherapy or radiation therapy for the current metastasectomy. 


\section{Discussion}

Dr Richard Fosburg (Del Mar, Calif). Dr Jaklitsch has described the outcome of 54 highly selected patients who underwent 154 operations in a 10-year interval at one institution. One half of these patients underwent a third metastasectomy, and the authors conclude that subsequent metastasectomies may not be warranted. This subset represents approximately $15 \%$ of all patients undergoing metastasectomy during the time frame, and the authors have provided us with 3 statistical predictors of survival.

I have trouble accepting the idea that metastasectomy is preserving control, rather than achieving control, but that can be discussed later.

Dr Jaklitsch, your predictors are thoracic control, the need for only 2 metastasectomies, and sustained absence of recurrent disease. From 1965 to the present, more than 450 articles on pulmonary metastasectomy have been published. That may be a bellwether that we have trouble determining what to do with this particular problem. Interestingly, in 1998 and 1999, a paper on this subject has been presented at the meetings of The American Association for Thoracic Surgery, The Society of Thoracic Surgeons, and The Western Thoracic Surgical Association. In 1947, John Alexander and Cameron Haight were the first to attempt to establish criteria for pulmonary resection. They had only a chest radiograph, and their dilemma was that they did not know whether a tumor appearing in the lung was a new primary or a metastasis. Therefore, they believed it reasonable to operate on all of them. The setting has changed. Now, 50 years later, we have the outcome of a pulmonary metastasectomy registry, which was begun in 1991, and you have referred to that registry. The contributors to the registry defined certain categories to use as their prognostic groups as well, and Pastorino, who was writing for the committee, had accrued 5206 cases and affirmed that complete removal of all metastatic deposits was associated with long-term survival. Therefore, we do have an objective.

In the groupings that the registry created, it was important that resectability always be present if prognosis was to be accurate. The absence of resectability is basically what determines whether you carry on. All retrospective analyses of this particular issue are characterized by a very similar fault, and that is that the true extent of disease is not known. Consequently, I think that the future will portend that we will do much better in understanding which candidates should undergo this procedure. I am referring to the evolution of positron emission tomography, using 18-fluorodeoxyglucose as one method of identifying other metastases. In 1998, the Health Care Financing Administration approved positron emission tomographic scanning for evaluation of metastatic disease in lung cancer, and beginning July 1, 1999, they extended the use of positron emission tomographic scanning for malignant melanoma, lymphoma, and colorectal cancer. At least we are moving in the right direction. Old agents such as technetium 99 will be replaced by newer agents, among which are 18-fluorothymidine, F18-tagged sodium fluoride, and labeled markers like carcinogenic embryonic antigen. Consequently, I believe the future is going to be different. The next analysis of this procedure may give us greater insight as to how to best handle metastases.

You indicated that you grouped those staged patients as 1 operation even though they may have had multiple operations to achieve clearance. Did any of those patients who were staged in the 3-month interval have new lesions develop during the interval between the operations?

Dr Jaklitsch. No. Only about 5 patients had a staged thoracotomy approach, about $11 \%$ of the entire group, and none of them had new metastases within the interval between operations. We do routinely repeat the CT scan before the second thoracotomy.

Dr Fosburg. The Memorial people have told us that the best way to determine a pulmonary metastasis is palpation by the operating surgeon. They say that many lesions are missed. That brings up the issue of how and when we do scans, at what level we do cuts, and what size lesions we are detecting. You indicated that VATS had been used in certain patients. Did any of the patients undergoing VATS also have further recurrences?

Dr Jaklitsch. Yes. The VATS operations cluster at the second and the third attempts, so quite a number of those procedures were followed by recurrence. We tested the use of a VATS incision on univariate analysis to see whether that was a predictor of recurrence and disease-free status and found no association.

This is an area of interest throughout the country, and many thoracic surgeons are being polarized regarding whether or not VATS is an appropriate operation to perform for metastasectomy. On one hand are McCormick and Martini's data, which show that about $43 \%$ of patients have undetected metastases that can be found at open thoracotomy. On the other hand is Putnam and Roth's information that the median sternotomy versus unilateral thoracotomy had no difference in ultimate survival of the patient. Their information suggests that lesions that are too occult to be detected radiographically, but can only be palpated by the surgeon's hand, may not be clinically important. I hope this area will be answered by the current Cancer and Leukemia Group B trial of VATS metastasectomy versus open thoracotomy, sponsored by the National Cancer Institute.

Dr Fosburg. In those patients who were identified to have a recurrence, you indicated that a helical CT scan was done generally within 6 weeks. Your data indicate that the median interval between the second and third procedures was about 12 months. Do you think 6 weeks is appropriate timing for the CT scan? Are you comfortable with that short interval to look at a process that seems to be extended over a longer interval?

Dr Jaklitsch. That is a very valid criticism. We have come to the realization that we are never free from the issue of recurrence in metastasectomy patients. In patients who we believe have no evidence of disease, we repeat the CT scan about 3 times a year, or every 4 months. It usually takes more than the first recurrence detected on the CT scan to convince us to reoperate. We usually repeat that CT scan between 6 
weeks and 3 months to judge the rate of progression. Now, many of the patients whose disease has become technically unresectable have had a blossoming during that interval, with the development of numerous lesions.

Dr Fosburg. The median size of the largest metastasis excised at each subsequent surgical procedure sequentially increased, as might be expected from a logarithmic growth curve. Were calculations of tumor doubling times used in any way to assist in your decisions?

Dr Jaklitsch. No. We do not prospectively use tumor doubling time. We tried to recreate it for this manuscript, but we were only able to generate it for about $25 \%$ of our patients.

Online-www.aats.org

Now you can get The Journal of Thoracic and Cardiovascular Surgery online.

The Journal online brings you faster delivery time, easy searching of current and back issues,

links to PubMed, AATS, WTSA, and other important sites, and more.

Visit the Journal online today.

\section{Receive tables of contents by e-mail}

To receive the tables of contents by e-mail, sign up through our Web site at http://www.mosby.com/jtcvs

Choose E-mail Notification

Simply type your e-mail address in the box and click the Subscribe button.

Alternatively, you may send an e-mail message to majordomo@mosby.com.

Leave the subject line blank and type the following as the body of your message: subscribe jtcvs_toc

You will receive an e-mail to confirm that you have been added to the mailing list. Note that TOC e-mails will be sent out when a new issue is posted to the Web site. 